\title{
Symmetrization of Feature Points in 2-D Images ${ }^{\#}$
}

\author{
Alexander Karkishchenko ${ }^{*}$, Valeriy Mnukhin ${ }^{1}$
}

Accepted $15^{\text {th }}$ August 2014

\begin{abstract}
In this work, we consider the symmetrization problem, that is the problem to obtain more accurate information about location of points based on a priori knowledge of their symmetries. Methods to solve the symmetrization problem with respect to vertical and inclined axes of reflectional symmetry are considered jointly with the more general symmetrization with respect to an indefinite reflection axis. Then the case of rotational symmetry is considered. The methods produce the minimal deformation that enhances approximate symmetries present in a given arrangement of points.
\end{abstract}

Keywords: Symmetrization, reflectional symmetry, rotational symmetry, feature points, biometrical identification.

\section{Introduction}

Symmetry is a central concept in many natural and man-made objects and plays a crucial role in visual perception, design and engineering. Several recent efforts in shape analysis have focused on detecting symmetries in 2-D and 3-D shapes [1]-[3]. Numerous applications have successfully utilized this type of information, e.g., for model reduction [3], scan completion [4], segmentation [5], shape matching [1], etc. In many cases lowlevel symmetry analysis is based on investigations of so-called feature points, whose exact meaning depends on the resolving problem.

One of the most common problems, where methods of "refinement by symmetry" can be efficiently used, is the biometrical identification, when the correct location of feature points is crucial. In particular, the accuracy of human face detection and recognition strongly depends on the measurement precision of pupils of eyes location [6]. Then the fact of near, but imperfect, reflective symmetry of human full-faces can be used to improve the accuracy [7]. In fact, usually methods of human face detection are based on the position analysis of several dozens feature points, which are either coupled in pairs, symmetric with respect to a vertical axis, or situated in the axis.

In this work, we present several methods to obtain more accurate information about location of feature points, based on a priori knowledge of their symmetry. Note that positions of points in images are always known with some drift that depends on such factors as the image quality, noise levels in a vicinity of the points, the processing algorithm, and so on. As a result, evaluated coordinates could fail the symmetry conditions even for those points, which are in fact symmetric. So it is reasonable to use the information about symmetry to specify positions of feature points. Besides, the symmetrization itself should be done with minimal deformation of points positions that enhances

\footnotetext{
. 'Southern Federal University, 105/42 Bolshaya Sadovaya Str., Rostovon-Don, 344006, Russia

* Corresponding Author: Email: karkishalex@gmail.com

${ }^{\text {\#}}$ This paper has been presented at the International Conference on Advanced Technology\&Sciences (ICAT'14) held in Antalya (Turkey), August 12-15, 2014.
}

approximate symmetries present in a given arrangement of points. The presented methods produce such optimal arrangements of feature points under reflectional and rotational symmetries.

\section{Symmetrization with Respect to a Vertical Axis}

Let $P=\left\{p_{1}, \ldots, p_{n}\right\}$ be the set of all feature points given by their coordinates $p_{k}=\left(x_{k}, y_{k}\right)$. Assume that the reflection symmetry axis coincides with the coordinate axis $O y$ and that the feature points are ordered in such a way that the points $P_{R}=\left\{p_{1}, \ldots, p_{m}\right\}$ are in the right half-plane, the corresponding points $P_{L}=\left\{p_{m+1}, \ldots, p_{2 m}\right\}$ are in the left half-plane, and the rest of points $P_{O}=\left\{p_{2 m+1}, \ldots, p_{n}\right\}$ are situated in the axis $O y$.

The situation is illustrated by Fig. 1, whose left hand part demonstrates a set of feature points, the central part shows its partition into classes $P_{R}, P_{L}, P_{O}$, and the right hand part shows the feature points after symmetrization.

We associate with the ordered set $P$ the following $2 n$ dimensional vector:

$\left(p_{1}, p_{2}, \ldots, p_{n}\right) \leftrightarrow\left(x_{1}, \ldots, x_{n}, y_{1}, \ldots, y_{n}\right)^{T}=X$

Evidently, all such vectors form the vector space $R^{2 n}$. Taking into account the partition into the classes $P_{R}, P_{L}, P_{O}$, note that if the positions of all the feature points are precisely known, the following conditions must be satisfied:

$$
\begin{array}{ll}
x_{i}=-x_{m+i}, & i=1, \ldots, m ; \\
y_{i}=y_{m+i}, & i=1, \ldots, m ; \\
x_{i}=0, & i=2 m+1, \ldots, n .
\end{array}
$$

It is easy to check that the set of all vectors in $R^{2 n}$ under these conditions form an $n$-dimensional subspace $R_{S y m} \subset R^{2 n}$.

Note that the "symmetrization" of a given arrangement $P$ of feature points means finding the vector $X_{s} \in R_{S y m}$ that is the best approximation of $X$ by the Euclidean norm:

$$
X_{s}=\arg \min _{Z \in R_{S s m}}\|Z-X\|
$$

Thus, $X_{s}$ is the orthogonal projection of $X$ onto the subspace 
$R_{\text {Sym }}$.

To find the projection $X_{s}$, let $Q$ be the projection matrix onto the subspace $R_{S y m}$, and let $A$ be a $(2 n \times n)$-matrix with $R_{S y m}$ as its column space, so that columns of $A$ form a basis for $R_{S y m}$.

Then, since $A$ is a matrix with linearly independent columns, we know [8], that $Q=A A^{+}=A\left(A^{T} A\right)^{-1} A^{T}$, where $A^{+}$is the pseudoinverse of $A$, and so $X_{s}=Q X$.

To complete the solution, note that

$A^{T}=\left(\begin{array}{cccccc}I_{m} & -I_{m} & 0 & 0 & 0 & 0 \\ 0 & 0 & 0 & I_{m} & I_{m} & 0 \\ 0 & 0 & 0 & 0 & 0 & I_{n-2 m}\end{array}\right)$

and so $Q=\frac{1}{2} I_{2 n}+\frac{1}{2}\left(\begin{array}{cc}-S & 0 \\ 0 & S\end{array}\right)$, where $S$ is the following $n \times n$ -

matrix:
$S=\left(\begin{array}{ccc}0 & I_{m} & 0 \\ I_{m} & 0 & 0 \\ 0 & 0 & I_{n-2 m}\end{array}\right)$

Hence, for the vertical reflection axis the symmetrization can be easily performed by producing simple operations over coordinates of feature points. Namely, to symmetrize a pair of points $p_{i} \in P_{R}$ and $p_{m+i} \in P_{L}$, we need to take a pair of points with coordinates

$$
\left( \pm \frac{\left|x_{i}\right|+\left|x_{m+i}\right|}{2}, \frac{y_{i}+y_{m+i}}{2}\right) \text {. }
$$

To symmetrize a point from $P_{O}$, we need to zeroize its $x$ coordinate, and leave its $y$-coordinate without changes.
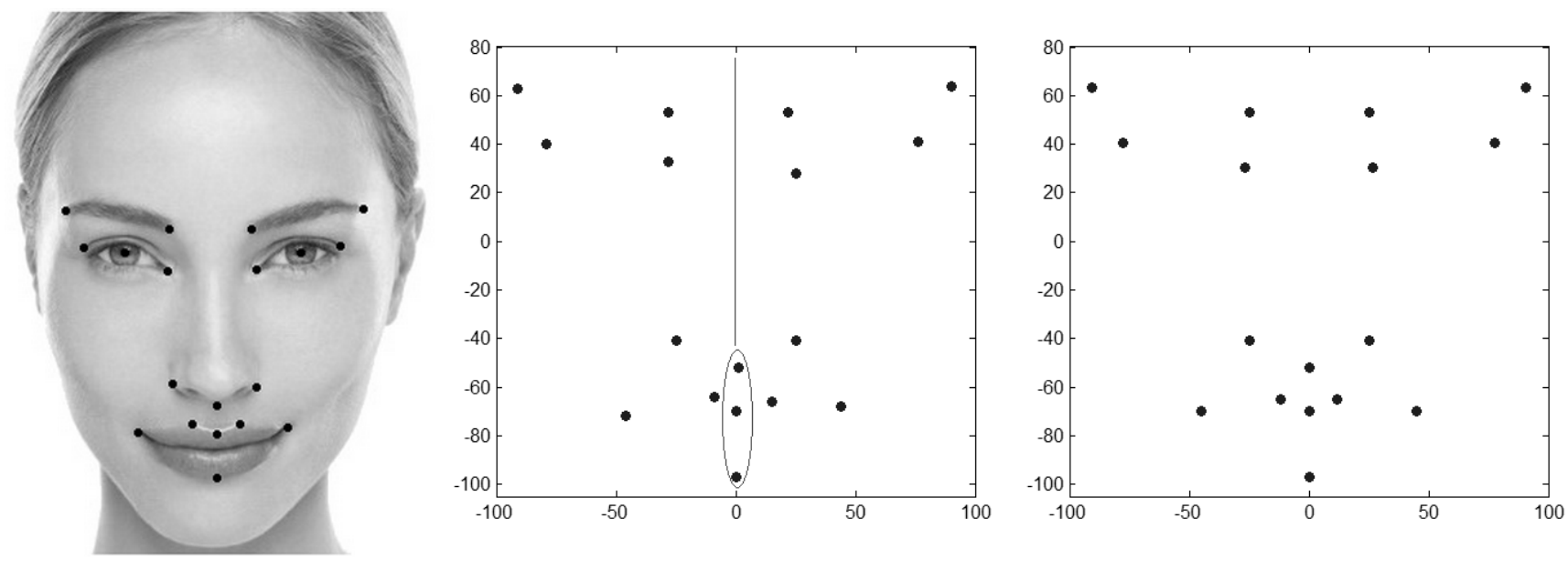

Fig.1. Symmetry with respect to the vertical axis $O y$.

\section{Symmetrization with Respect to an Arbitrary Axis}

Now, let the reflection axis be defined by the equation $y=a x+b$ , (where $a \neq 0$ ), as it is shown in Fig. 2. Then the symmetrization problem can be solved by switching to a new coordinate system $\left(O^{\prime} x^{\prime} y^{\prime}\right)$. Namely, assume that the $y^{\prime}$-axis of the new system coincides with the reflection axis, the origin $O^{\prime}$ is in the point, where $y^{\prime}$-axis crosses $O y$, and $O^{\prime} x^{\prime}$ is orthogonal with $O^{\prime} y^{\prime}$ in such a way that $\left(O^{\prime} x^{\prime} y^{\prime}\right)$ is a "right-handed" system.

Let $(x, y)$ be coordinates of an arbitrary point in the "old" $(O x y)$-system, and let $\left(x^{\prime}, y^{\prime}\right)$ be coordinates of the same point in the "new" $\left(O^{\prime} x^{\prime} y^{\prime}\right)$-system. Then

$$
x^{\prime}=x \cos \varphi+(y-b) \sin \varphi
$$$$
y^{\prime}=-x \sin \varphi+(y-b) \cos \varphi \text {, }
$$

where the values of $\cos \varphi$ and $\sin \varphi$ follow from the condition $\cot \varphi=-a \neq 0$, so that

$$
\sin \varphi=\frac{1}{\sqrt{a^{2}+1}}, \quad \cos \varphi=-\frac{a}{\sqrt{a^{2}+1}} .
$$

Just as above, we consider the vector

$$
X=\left(x_{1}, \ldots, x_{n}, y_{1}, \ldots, y_{n}\right)^{T} \in R^{2 n},
$$

formed by coordinates of the feature points with respect to the "old" $(O x y)$-system, and the vector $X^{\prime}$ of coordinates of the same points with respect to $\left(O^{\prime} x^{\prime} y^{\prime}\right)$. These vectors are related by $X^{\prime}=R(X-b K)$, where $K=(0, \ldots, 0,1, \ldots, 1)^{T}$, and

$R^{T}=\left(\begin{array}{cc}\cos \varphi \cdot I_{n} & \sin \varphi \cdot I_{n} \\ -\sin \varphi \cdot I_{n} & \cos \varphi \cdot I_{n}\end{array}\right)$ 


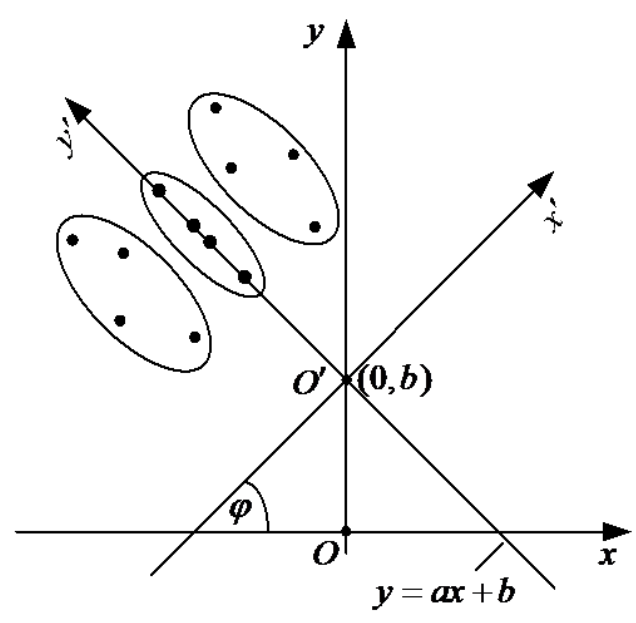

Fig. 2. Symmetrization with respect to an arbitrary axis.

It converts the symmetrization with respect to an arbitrary axis into the previously solved problem. Indeed, we may symmetrize $X^{\prime}$ in the same way, as it has been done in the previous section, and then return the symmetrized vector $X_{s}^{\prime}$ back into the $(O x y)$ system:

$$
X_{s}=R X_{s}^{\prime}+b K=R^{T}\left(Q R^{T}(X-b K)\right)+b K
$$

or

$$
X_{s}=G X+b H K \text {, where } G=R Q R^{T} \text { and } H=I-R Q R^{T}
$$

Now, it as an easy matter to work out that

$$
G=\frac{1}{2} I_{2 n}+\frac{1}{2}\left(\begin{array}{rr}
-\cos 2 \varphi \cdot S & \sin 2 \varphi \cdot S \\
\sin 2 \varphi \cdot S & \cos 2 \varphi \cdot S
\end{array}\right)
$$

the expression for $H$ is similar.

\section{Symmetrization with Respect to an Unknown Axis}

Assume now, that the parameters $a$ and $b$ of a reflection symmetry axis are unknown, though the existence of such axis follows from the nature of the problem under solution. In this section we use the previously developed methods to determine the parameters in such a way that symmetrization could be achieved by the minimal deformation. For this we need the useful notations:

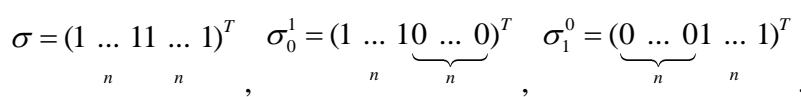

So let the reflection axis be defined by $y=a x+b$, where $a=-\cot \varphi \neq 0$ and $b$ are undefined parameters. The corresponding optimization problem is

$$
\left\|A Y-R^{T}(\varphi)\left(X-b \sigma_{1}^{0}\right)\right\|^{2} \stackrel{Y, \varphi, b}{\longrightarrow} \min ,
$$

where $A Y=Z$ is the resulting vector of coordinates of symmetrized points. In other words, to achieve the optimal symmetrization we need to find the parameters $\varphi, b$, and the minimizing vector $Y$.

Let $F(Y, \varphi, b)$ be the expression under the minimization. Writing it in a matrix form and using the minimization criteria, we would get the following equations: $\frac{\partial F(Y, \varphi, b)}{\partial Y}=0 \quad \frac{\partial F(Y, \varphi, b)}{\partial \varphi}=0 \quad \frac{\partial F(Y, \varphi, b)}{\partial b}=0$.

A series of appropriate calculations and transformations produce the system

$$
\left\{\begin{array}{l}
A Y=Q R^{T}(\varphi)\left(X-b \sigma_{1}^{0}\right), \\
\left(X-b \sigma_{1}^{0}\right)^{T} \frac{d R(\varphi)}{d \varphi} A Y=0, \\
\left(\sigma_{1}^{0}\right)^{T} R(\varphi) A Y=\left(\sigma_{1}^{0}\right)^{T}\left(X-b \sigma_{1}^{0}\right) .
\end{array}\right.
$$

The first (matrix) equation gives us the symmetrized vector in relation with $\varphi$ and $b$. The last two scalar equations can be used to find the parameters $\varphi$ and $b$ of the reflection axis.

To write down the final solution, assume that the vector $X$ is partitioned into the "centralized" blocks $X=\left(\dot{x}_{1}^{T} \dot{x}_{2}^{T} \dot{x}_{3}^{T} \dot{y}_{1}^{T} \dot{y}_{2}^{T} \dot{y}_{3}^{T}\right)$ where $\dot{x}_{i}=x_{i}-x_{a v} e, \dot{y}_{i}=y_{i}-y_{a v} e, i=1,2,3, e$ is the vector of 1 's of an appropriate dimension, and $x_{a v}=\frac{1}{n} \sum_{i=1}^{n} x_{i}$ and $y_{a v}=\frac{1}{n} \sum_{i=1}^{n} y_{i}$ are averages of $x$ - and $y$-coordinates of the evaluated feature points. Then, after some tedious transformations of the last two equations of the system, the next result follows:

$$
\begin{aligned}
& \operatorname{tg} 2 \varphi=\frac{\left(\dot{x}_{1}, \dot{y}_{2}\right)+\left(\dot{x}_{2}, \dot{y}_{1}\right)+\left(\dot{x}_{3}, \dot{y}_{3}\right)}{\left(\dot{x}_{1}, \dot{x}_{2}\right)-\left(\dot{y}_{1}, \dot{y}_{2}\right)+\frac{1}{2}\left(\dot{x}_{3}, \dot{x}_{3}\right)-\frac{1}{2}\left(\dot{y}_{3}, \dot{y}_{3}\right)}, \\
& b=y_{a v}+x_{a v} \cot \varphi
\end{aligned}
$$

Substitute the evaluated parameters $\varphi$ and $b$ into the first equation of the system, we can find the symmetrization $A Y=Z$ of the original vector $X$. As the last step of the solution, the symmetrized vector should be transformed into the original "old" coordinate system.

An example of symmetrization with an unknown axis is given in Fig. 3. Distorted feature points with respect to the original axis are shown in its upper right-hand part, while its lower left part shows the reconstructed axis and the result of symmetrization with respect to it. The two axes are compared in the lower righthand part of Fig. 3.

\section{Rotational Symmetrization of Feature Points}

Let $p=\left(\begin{array}{llll}p_{1} & p_{2} & \ldots & p_{n}\end{array}\right)^{T}$ be the vector of all feature points $p_{k}=\left(\begin{array}{ll}x_{k} & y_{k}\end{array}\right)^{T}, k=1,2, \ldots, n$. Assume that it is known a priori that this set of points is rotational symmetric and ordered in such a way that $p_{k+1}=R_{\varphi} p_{k}$ for all $k=1,2, \ldots, n-1$, where $R_{\varphi}$ is the rotation operator with the angle $\varphi=2 \pi / n$. In particular, $p_{k}=R_{\varphi}^{k-1} p_{1}$. Just as above, associate with $p$ the following vector $X$,

$p=\left(\begin{array}{llll}p_{1} & p_{2} & \ldots & p_{n}\end{array}\right)^{T} \leftrightarrow\left(x_{1} y_{1} x_{2} y_{2} \ldots x_{n} y_{n}\right)^{T}=X$

and note that all such $X$ fill the space $R^{2 n}$. Using matrices, the rotation symmetry condition can be written as 
$\left(\begin{array}{l}x_{k+1} \\ y_{k+1}\end{array}\right)=\left(\begin{array}{cc}\cos \varphi & -\sin \varphi \\ \sin \varphi & \cos \varphi\end{array}\right)\left(\begin{array}{l}x_{k} \\ y_{k}\end{array}\right)=\left(\begin{array}{cc}\cos k \varphi & -\sin k \varphi \\ \sin k \varphi & \cos k \varphi\end{array}\right)\left(\begin{array}{l}x_{1} \\ y_{1}\end{array}\right)$

for all $k=1,2, \ldots, n-1$, or simultaneously for all points as

$X=\left(\begin{array}{c}p_{1} \\ p_{2} \\ \ldots \\ p_{n}\end{array}\right)=\left(\begin{array}{c}I \\ R_{\varphi} \\ \ldots \\ R_{(n-1) \varphi}\end{array}\right) p_{1} \quad R_{\varphi}=\left(\begin{array}{cc}\cos \varphi & -\sin \varphi \\ \sin \varphi & \cos \varphi\end{array}\right)$ and

$p_{k}=\left(\begin{array}{l}x_{k} \\ y_{k}\end{array}\right)$

The set of all such vectors $X$ under this condition form a 2-
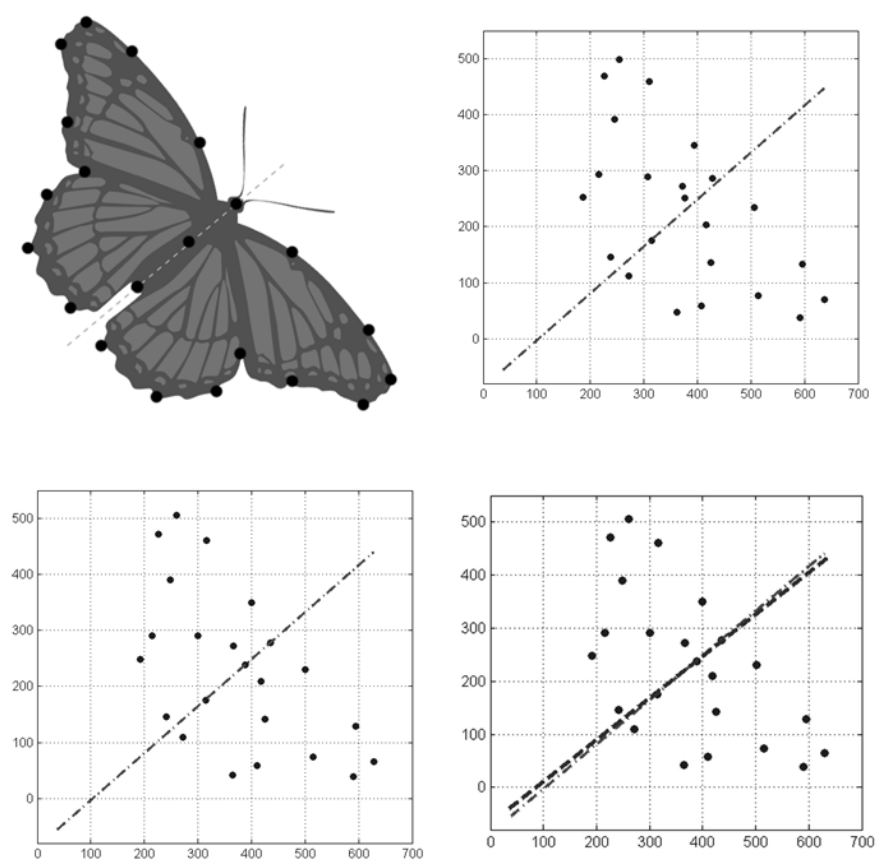

Fig. 3. Example of symmetrization with an unknown axis.

Now, assume that $X$ corresponds to the given arrangement of feature points, so that $X \notin R_{S y m}$. The symmetrization means finding the vector $X_{S}$ in $R_{S y m}$ that approximates $X$ in the best way: $X_{S}=\arg \min \|Z-X\|$. Just as for the case of reflectional symmetry, $X_{S}$ is is the orthogonal projection of $X$ onto the subspace $R_{S y m}$, and so $X_{S}=A\left(A^{T} A\right)^{-1} A^{T} X=Q X$.

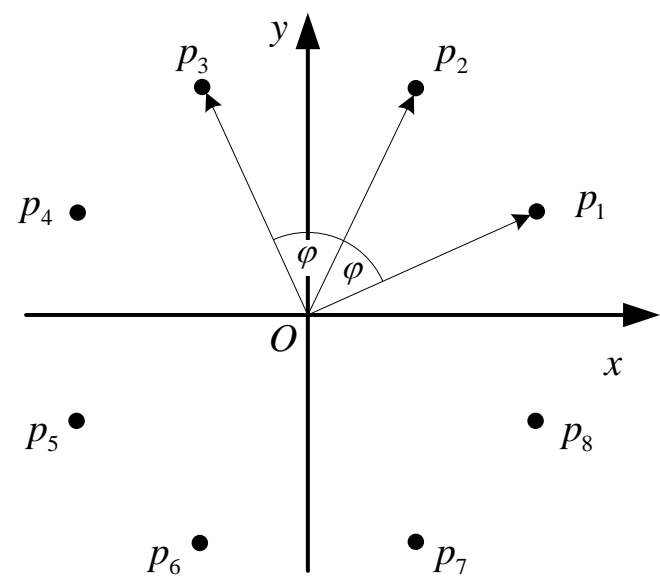

dimensional subspace $R_{S y m}$ in $R^{2 n}$, spanned by columns of the matrix

$$
A=\left(\begin{array}{c}
I \\
R_{\varphi} \\
\cdots \\
R_{(n-1) \varphi}
\end{array}\right)
$$


$A\left(A^{T} A\right)^{-1} A^{T}=\frac{1}{n}\left(\begin{array}{cccc}I & R_{(n-1) \varphi} & \ldots & R_{\varphi} \\ R_{\varphi} & I & \ldots & R_{2 \varphi} \\ \ldots & \ldots & I & \ldots \\ R_{(n-1) \varphi} & R_{(n-2) \varphi} & \ldots & I\end{array}\right)$

To understand meaning of the gained result, note that

$X_{S}=A\left(A^{T} A\right)^{-1} A^{T} X=\left(\begin{array}{c}I \\ R_{\varphi} \\ \ldots \\ R_{(n-1) \varphi}\end{array}\right) \cdot \frac{1}{n}\left(\begin{array}{llll}I & R_{\varphi}^{T} & \ldots & R_{(n-1) \varphi}^{T}\end{array}\right)\left(\begin{array}{c}p_{1} \\ p_{2} \\ \ldots \\ p_{n}\end{array}\right)=$

$=\left(\begin{array}{c}I \\ R_{\varphi} \\ \ldots \\ R_{(n-1) \varphi}\end{array}\right) \cdot \frac{1}{n}\left[I p_{1}+R_{\varphi}^{T} p_{2}+R_{2 \varphi}^{T} p_{3}+\ldots+R_{(n-1) \varphi}^{T} p_{n}\right]=$

$=\left(\begin{array}{c}I \\ R_{\varphi} \\ \cdots \\ R_{(n-1) \varphi}\end{array}\right) \cdot \frac{1}{n} \sum_{k=1}^{n} R_{(k-1) \varphi}^{T} p_{k}$

The term $R_{(k-1) \varphi}^{T} p_{k}=R_{(k-1) \varphi}^{-1} p_{k}$ may be considered as coordinates of the point ${ }^{p_{k}}$ rotated with the angle ${ }^{(k-1) \varphi}$ clockwise and so situated in the vicinity of the point ${ }^{p_{1}}$. Such "backward" rotations of $p_{1}, p_{2}, \ldots, p_{n}$ would form a "cloud" of points around $p_{1}$. So the second factor in the expression for $X_{s}$ above may be considered as the "center of the cloud" with average coordinates. Multiplication by the block matrix produce the optimal rotational symmetrized arrangement of feature points.

\section{Conclusion}

The considered problem of symmetrization of characteristic points relative to the axial and rotational symmetry has numerous applications, since it is the simplest and most common type of symmetry.

We have shown how to symmetrize feature points of an image with respect to vertical and inclined axes of reflectional symmetry and considered the more general symmetrization with respect to an indefinite reflection axis. Together with it the case of rotational symmetry is considered. It is worth to mention that all the methods produce the minimal deformation that enhances approximate symmetries present in a given arrangement of points.

However, there are also other types of symmetry - dihedral, translational and others, that can be met in the tasks related to image processing. The particular interest is the symmetrization under affine distortions, which occur in most real developments. A rigorous mathematical solution of these problems may not be easy, but it will provide additional opportunities for high-quality image processing.

\section{Acknowledgements}

The authors gratefully acknowledge partial support of the reported study by RFBR, research projects No. 13-07-13112 ofi_m_RzhD and No. 11-07-00591.

\section{References}

[1] J. Podolak, P. Shilane, J. Giesen, M. Gross, and L. Guibas, "Example-based 3D scan completion," in Proc. Symposium on Geometry Processing, pp 23-32, 2005.

[2] A. Martinet, C. Soler, N. Holzschuch, and F. Sillion, "Accurate detection of symmetries in 3D shapes," ACM Trans. Graph, vol. 25, \# 2, pp. 439-464, 2006.

[3] N. J. Mitra, L. J. Guibas, and M. Pauly, "Partial and approximate symmetry detection for 3D geometry," ACM Trans. Graph, vol. 25, \# 3, pp. 560-568, 2006.

[4] S. Thrun, and B. Wegbreit, "Shape from symmetry," in Proc. Int. Conference on Computer Vision (ICCV), vol. 2, pp. 1824-1831, 2005.

[5] P. Simari, E. Kalogerakis, and K. Singh, "Folding meshes: Hierarchical mesh segmentation based on planar symmetry," in Proc. Symposium on Geometry Processing, 2006.

[6] A. N. Karkishchenko, and I. A. Grechukhin, "Statistical face recognition based on the geometry of feature points," (in Russian), in Proc. Large-Scale Systems Control, vol. 38, pp. 78-90, Moscow, 2012.

[7] A. N. Karkishchenko, and I. A. Grechukhin, "Localization of feature points based on the natural symmetries of images," (in Russian), in Proc. of the Conference "Intellectual Control Systems for Rail Transport," pp. 262265, Moscow, 2012.

[8] G. Strang, Linear Algebra and Its Applications. Thomson Brooks/Cole, 2006 